\title{
The Presence of Neural Stem Cells and Changes in Stem Cell-Like Activity With Age in Mouse Spiral Ganglion Cells In Vivo and In Vitro
}

\author{
Byoung-San Moon ${ }^{1,2} \cdot$ Aswathy Ammothumkandy ${ }^{1}$ Naibo Zhang ${ }^{1} \cdot$ Lei Peng $^{1} \cdot$ Albina lbrayeva $^{1} \cdot$ Maxwell Bay $^{1}$ \\ Athira Pratap ${ }^{A} \cdot$ Hong Ju Park ${ }^{3, *} \cdot$ Michael Anthony Bonaguidi' ${ }^{1}$ Wange $\mathrm{Lu}^{1, *}$ \\ ${ }^{I}$ Department of Stem Cell Biology and Regenerative Medicine, Broad Center for Regenerative Medicine and Stem Cell Research and \\ ${ }^{2}$ Department of Neurosurgery, Keck School of Medicine of the University of Southern California, Los Angeles, CA, USA; \\ ${ }^{3}$ Department of Otorhinolaryngology-Head and Neck Surgery, Asan Medical Center, University of Ulsan College of Medicine, Seoul, Korea
}

Objectives. Spiral ganglion neurons (SGNs) include potential endogenous progenitor populations for the regeneration of the peripheral auditory system. However, whether these populations are present in adult mice is largely unknown. We examined the presence and characteristics of SGN-neural stem cells (NSCs) in mice as a function of age.

Methods. The expression of Nestin and Ki67 was examined in sequentially dissected cochlear modiolar tissues from mice of different ages (from postnatal day to 24 weeks) and the sphere-forming populations from the SGNs were isolated and differentiated into different cell types.

Results. There were significant decreases in Nestin and Ki67 double-positive mitotic progenitor cells in vivo with increasing mouse age. The SGNs formed spheres exhibiting self-renewing activity and multipotent capacity, which were seen in NSCs and were capable of differentiating into neuron and glial cell types. The SGN spheres derived from mice at an early age (postnatal day or 2 weeks) contained more mitotic stem cells than those from mice at a late age.

Conclusion. Our findings showed the presence of self-renewing and proliferative subtypes of SGN-NSCs which might serve as a promising source for the regeneration of auditory neurons even in adult mice.

Keywords. Spiral Ganglion; Neural Stem Cells; Deafness; Hearing Loss; Cochlea

\section{INTRODUCTION}

- Received June 7, 2018

Revised August 8, 2018

Accepted August 10, 2018

- Corresponding author: Hong Ju Park

Department of Otorhinolaryngology-Head and Neck Surgery, Asan Medical

Center, University of Ulsan College of Medicine, 88 Olympic-ro 43-gil,

Songpa-gu, Seoul 05505, Korea

Tel: +82-2-3010-3700, Fax: +82-2-489-2773

E-mail: dzness@amc.seoul.kr

\section{- Co-Corresponding author: Wange Lu}

Department of Stem Cell Biology and Regenerative Medicine, Broad Center for Regenerative Medicine and Stem Cell Research, Keck School of Medicine of the University of Southern California, Los Angeles, CA 90033, USA

Tel: +1-323-442-1618, Fax: +1-323-442-4040

E-mail: wangelu@usc.edu

*Hong Ju Park and Wange Lu contributed equally to this study.
Sensorineural hearing loss is caused by the loss of the cochlear hair cells with the consequential degeneration of spiral ganglion neurons (SGNs), which relay auditory information from hair cells to the brainstem. The degeneration of SGNs is seen in circumstances such as noise exposure and aging, and overexposure to intense sound can cause an acute loss of SGN peripheral nerve terminals and a delayed loss of SGNs over a period of months [1]. In a report studying the number of normal cochlear fibers in rat, their number in young adults (2-3 months) was reduced by $21 \%$ in adult ( 26.5 months) and $24 \%$ in oldest (35-36 months) mice [2]. Degeneration of the SGNs following hair cell loss, which leaves fewer neurons available for electrical stimulation, carries critical implications for cochlear implants (CIs) be-

Copyright $\odot 2018$ by Korean Society of Otorhinolaryngology-Head and Neck Surgery.

This is an open-access article distributed under the terms of the Creative Commons Attribution Non-Commercial License (http://creativecommons.org/licenses/by-nc/4.0)

which permits unrestricted non-commercial use, distribution, and reproduction in any medium, provided the original work is properly cited. 
cause they stimulate the spiral ganglion cells directly by bypassing the cochlear hair cells.

Recent advances in stem cell therapy and cellular reprogramming have developed several possibilities to induce endogenous regeneration or exogenous stem cell transplantation for replacing decreased SGNs to enhance hearing restoration in patients with CIs [3-5]. However, the lack of suitable donor cells and the low survival rate of the implanted cells are major obstacles to the successful implementation of exogenous transplantation [6]. Several studies have reported the identification of SGN-derived neural stem cells (SGN-NSCs) and their differentiation into neurons to replace damaged SGNs by the endogenous regeneration of the SGNs [7-10]. It was reported that SGNs from humans aged between 40 and 60 years old formed spheres that proliferated and differentiated into mature neurons and glial cells, but mouse studies have revealed the existence of sphere-forming SGN-NSCs only during the early postnatal period of up to 3 weeks $[8,9]$. Thus, it is still unclear how long the SGN-NSCs remain present in the cochlear modiolus.

In this study, we examined the expression of Nestin and Ki67 in sequentially dissected cochlear modiolar tissues from mice of different ages (from postnatal day [P1] to 24 weeks) as well as the isolation of sphere-forming stem cells from spiral ganglion cells and their differentiation into different cell types.

\section{MATERIALS AND METHODS}

\section{Experimental animals and ethical considerations}

The mice for the experiments were maintained on a C57BL/6 background. All of them were housed under a 12-hour lightdark cycle and had access to food and water ad libitum in a controlled animal facility. All of the animal experimental procedures were approved by the Institutional Animal Care and Use Committee of the University of Southern California (Protocol No. 11489) and the National Institute of Health.

\section{H I G H L I G G T T S}

- There were Nestin+/Ki67+spiral ganglion neurons (SGNs) even in 6-month-old mice.

- SGN spheres from young mice had more mitotic cells than those from old mice.

- SGNs have multipotent capacity of differentiating into neuron and glial cells.

- The number of neural stem cells (NSCs) in vivo decreased as a function of age.

- SGN-NSCs may serve as a source for the regeneration of SGNs even in old mice.
The isolation, culture, and propagation of sphere-forming stem cells from the early and late postnatal spiral ganglion

The mice (P1, $n>30 ; 2$ weeks, $n>30 ; 1$ month, $n>15 ; 3$ month, $\mathrm{n}>10 ; 6$ month, $\mathrm{n}>10$ ) were decapitated, after which the otic capsule was dissected out after removal of the brain and immersed in ice-cold phosphate-buffered saline (PBS; Invitrogen, Carlsbad, CA, USA). The bony otic capsule was opened and removed to visualize the membranous labyrinth of the cochlea. The cochlear duct was microdissected from the modiolus where the spiral ganglion resides. The isolated spiral ganglion cells were cultured in N2 medium containing basic fibroblast growth factor (bFGF; 20 ng/mL, PeproTech, Rocky Hill, NJ, USA) for 4 days to maintain stem cell characteristics and then evaluated for Nestin and Sox 2 expression. To induce differentiation, the cells were seeded and further cultured in bFGF depleted medium [11-13]. Neurosphere culturing was conducted as has been described previously [11-13].

\section{The antibodies and reagents}

The antibodies used in this study were anti-Ki67 (rabbit polyclonal 1:500; Abcam, Cambridge, MA, USA), anti-Nestin (mouse monoclonal 1:350; BD Biosciences, San Jose, CA, USA), anti-Sox2 (rabbit polyclonal 1:500, Abcam), anti-Tuj1 (mouse polyclonal 1:200; Covance, Princeton, NJ, USA), antiMAP2 (rabbit polyclonal 1:200; Chemicon, Temecula, CA, USA), and anti-GFAP (rabbit 1:500; Cell Signaling Technology, Danvers, MA, USA). Secondary antibodies were anti-rabbit Alexa Fluor 488-conjugated, anti-mouse Alexa Fluor 488-conjugated, anti-rabbit Alexa Fluor 555-conjugated, and anti-mouse Alexa Fluor 555-conjugated immunoglobulin G (1:200 dilution; Molecular Probes, Eugene, OR, USA). The protease inhibitor cocktail was from Roche Applied Science (Indianapolis, IN, USA).

\section{Immunohistochemistry and immunocytochemistry}

Immunohistochemistry and immunocytochemistry was conducted as has been described previously [11-13]. Briefly, postnatal mice (P1, 2 weeks old) were euthanized with carbon dioxide. Cochlear tissues were isolated from the head and fixed in $4 \%$ paraformaldehyde at $4^{\circ} \mathrm{C}$ overnight. Adult mice (4 weeks, 12 weeks, and 24 weeks old) were anesthetized with isoflurane and transcardially perfused with saline, followed by $4 \%$ paraformaldehyde. Temporal bones containing the inner ear were removed and fixed in $4 \%$ paraformaldehyde overnight, after which they were moved to a $4 \%$ solution of ethylenediaminetetraacetic acid for 5 days to decalcify the bone. Cochlear tissues were cryoprotected in 30\% sucrose, embedded and frozen in the Tissue Tek OCT compound, and sectioned at $20 \mu \mathrm{m}$ on a cryostat. For the immunocytochemistry, spiral ganglion NSCs cultured on coverslips were fixed with $4 \%$ paraformaldehyde/ PBS for 30 minutes and immunostained after permeabilization with $0.2 \%$ Triton X-100. The cryosections or cells grown on cov- 
erslips were incubated with one of the primary antibodies at $4^{\circ} \mathrm{C}$ overnight. Cells and tissues were washed three times in PBS and incubated with Alexa Fluor 488- or 555-conjugated immunoglobulin G secondary antibody (Molecular Probes) at room temperature for 1 hour. Nuclei were counterstained in $1 \mu \mathrm{g} / \mathrm{mL}$ DAPI (4', 6-diamidino-2-phenylindole; Boehringer Mannheim, Mannheim, Germany) for 10 minutes followed by exhaustive washing in distilled water. The images were visualized using confocal microscopy (LSM 700; Zeiss, Jena, Germany). For quantification of images, approximately 100 cells were counted in at least three randomly selected $400 \times$ fields, and the percentages of proliferating cells (Ki67- or Nestin-positive) and progenitor- and neural differentiation-marker expressing cells were determined. All data were presented as mean and standard deviation (SD).

\section{The quantitative polymerase chain reaction protocol}

The quantitative polymerase chain reaction (qPCR) was conducted as has been described previously $[11,13]$. The primer
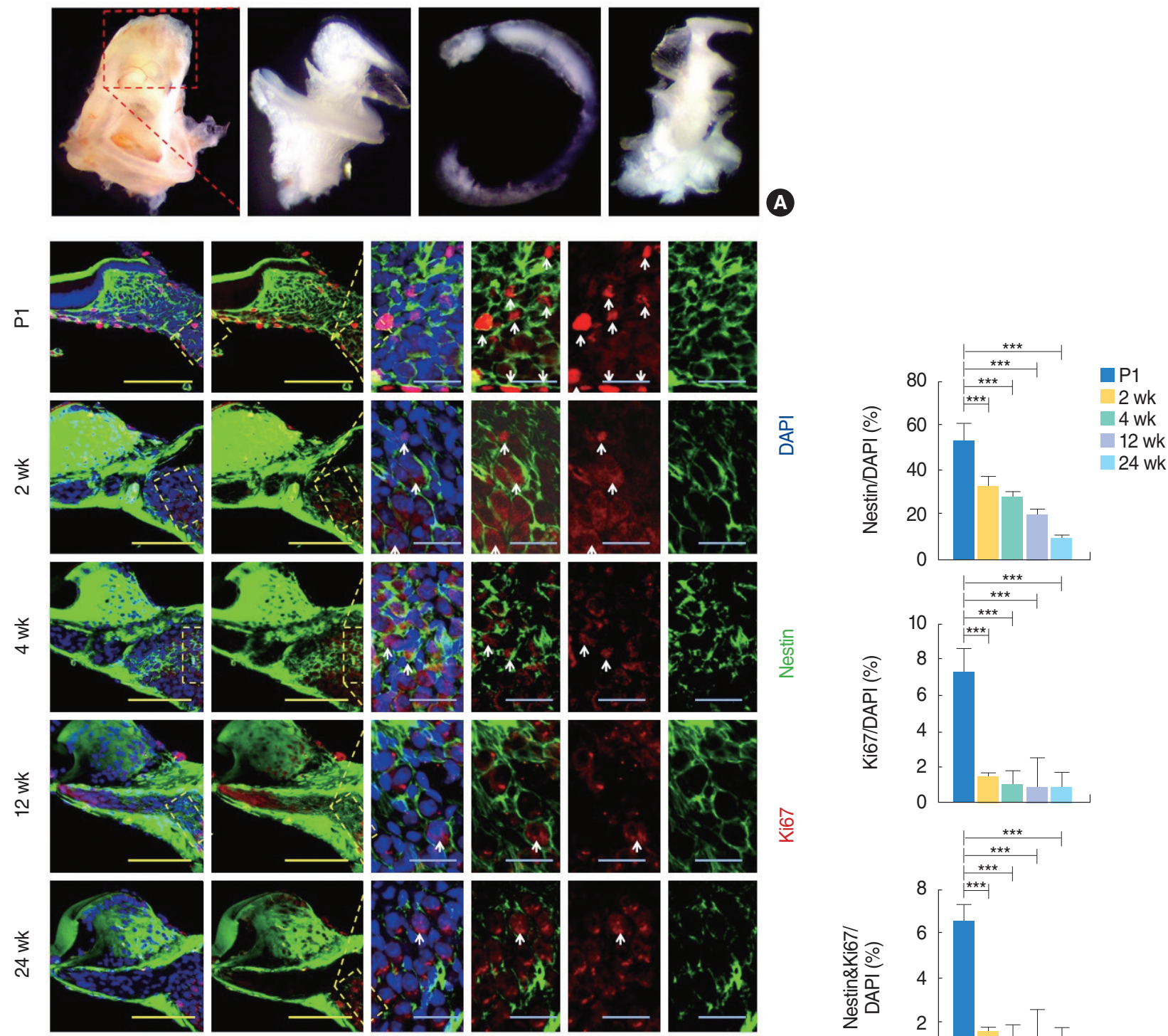

$100 \mu \mathrm{m}$

$-25 \mu \mathrm{m}$
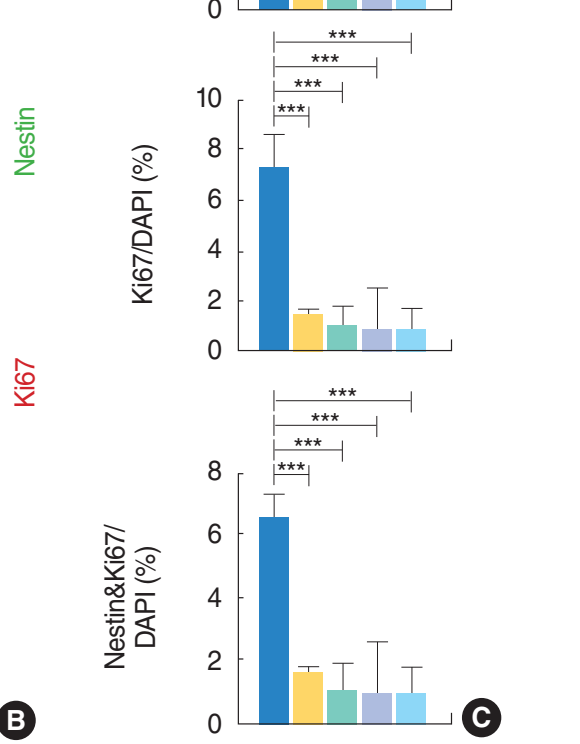

$\underline{\underline{\underline{0}}}$

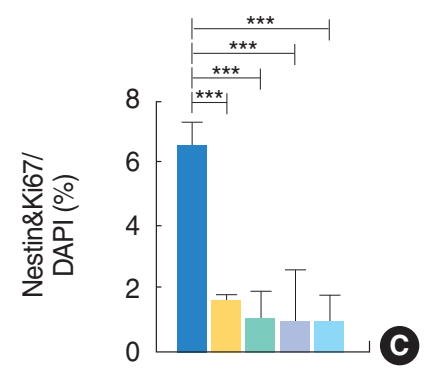

Fig. 1. Surgical dissection of the cochlea and sections for spiral ganglion neurons in postnatal mice as a function of age. (A) The bony shell of the cochlea (red box) was removed and then spiral cochlear tissue was removed, resulting in the modiolus containing spiral ganglion neurons. (B) Fixed coronal sections of the spiral ganglion neurons (yellow boxes) stained with antibodies against Nestin (green) and Ki67 (red). White arrows indicate Nestin and Ki67 double positive cells. (C) Quantification of the data shown in (B) (postnatal day 1 [P1], $n=4 ; 2$ weeks, $n=3 ; 4$ weeks, $n=3 ; 12$ weeks, $n=3$; and 24 weeks, $n=3$ ). All individual quantification data underlying (C) can be found in Supplementary Table 1. Values are presented as mean \pm standard deviation. Analysis of variance tests were performed to calculate significance $\left({ }^{\star * *} P<0.0001\right)$. DAPI, 4',6-diamidino-2-phenylindole. 
sets for the qPCR protocol are listed in Supplementary Table 2.

\section{Statistical analysis}

Statistical differences among the groups were analyzed using the Student $t$-test or analysis of variance (Tukey's multiple comparison test). The values obtained from at least three independent experiments were averaged and reported as the mean and SD. Any differences are indicated in the figures as follows: $P<0.05$, $P<0.005$, and $P<0.0005$. The $P$-value $<0.05$ was considered as statistically significant.
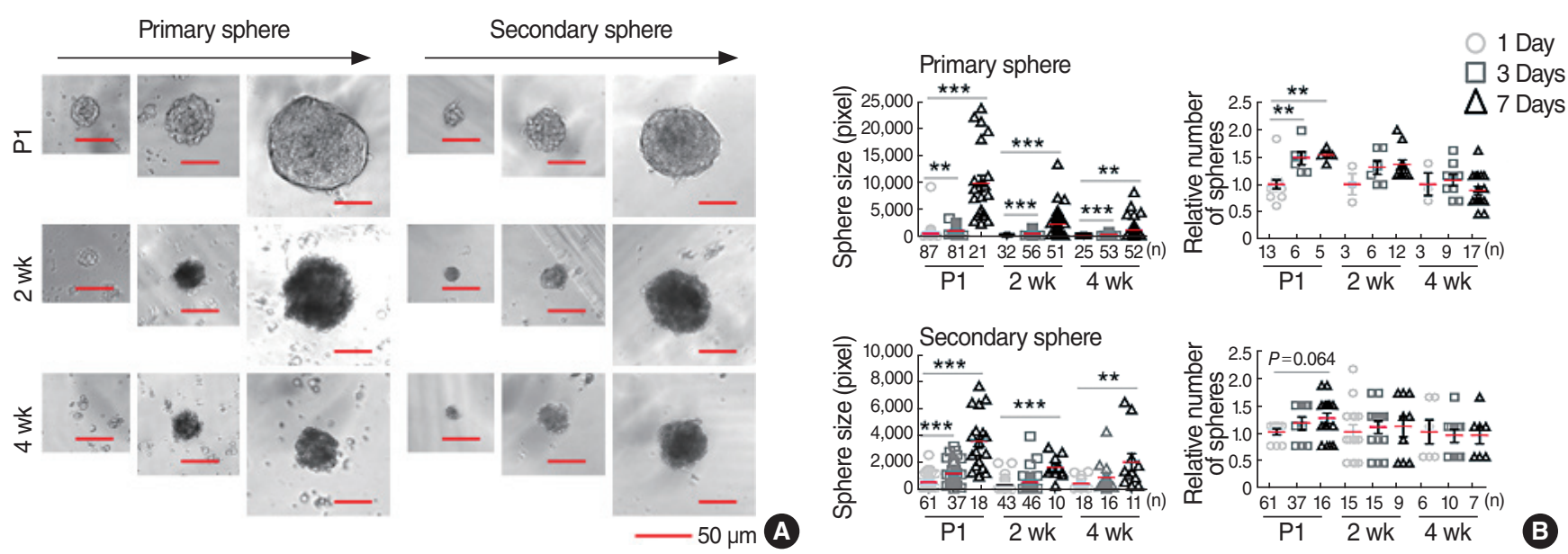
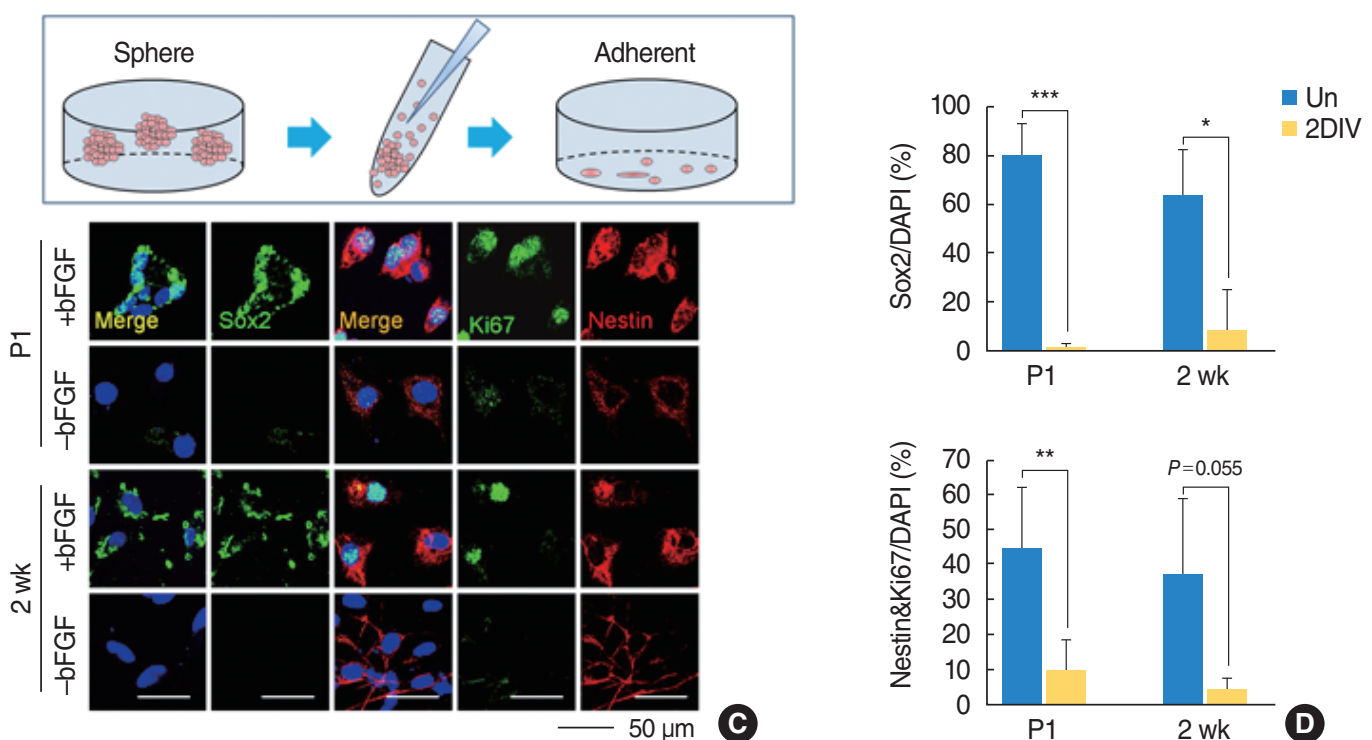

Fig. 2. Spiral ganglion cells exhibit self-renewing and proliferative activity. (A) Spiral ganglions were separated from mice of age postnatal day 1 (P1), 2 weeks, and 4 weeks, and sphere formation was induced for 7 days in vitro. (B) Quantification of the relative sizes and numbers of primary or secondary spheres in (A). The number of P1 spiral ganglion neuron-derived neural stem cell spheres formed during 1 day culture was normalized to 1 . Values are presented as mean \pm standard deviation. Analysis of variance tests were performed to calculate significance $\left({ }^{\star} P<0.01,{ }^{\star \star} P<0.001,{ }^{\star \star \star} P<0.0001\right)$. (C) P1 spiral ganglion cells dissociated from the spheres (upper) were grown and were subjected to immunofluorescence labeling using antibodies specific for Sox2 (green), Ki67 (green), and Nestin (red). (D) Quantification of anti-Sox2-positive (P1: +bFGF, $n=3$; -bFGF, $n=5$; 2 weeks: +bFGF, $n=3$; -bFGF, $n=4)$, or anti-Nestin and anti-Ki67 double-positive cells (P1: +bFGF, $n=6$; -bFGF, $\mathrm{n}=5 ; 2$ weeks: +bFGF, $\mathrm{n}=4$; -bFGF, $\mathrm{n}=3$ ) in (C). The $t$-tests were performed to calculate significance $\left({ }^{\star} P<0.05,{ }^{* \star} P<0.005,{ }^{* \star *} P<0.0005\right)$. bFGF, basic fibroblast growth factor; DAPI, 4’,6-diamidino-2-phenylindole; Un, undifferentiated; 2DIV, 2 days differentiation in vitro. 
$\sim 32 \%, \sim 27 \%, \sim 20 \%$, and $\sim 9 \%$ at P1, 2 weeks, 4 weeks, 12 weeks, and 24 weeks, respectively) with mouse age (Fig. 1B, C). Similarly, we observed a significant decrease in proliferation marker Ki-67-positive cells $(\sim 7.3 \%, \sim 1.5 \%, \sim 1 \%, \sim 1 \%$, and $\sim 0.9 \%$ at P1, 2 weeks, 4 weeks, 12 weeks, and 24 weeks, respectively) and Nestin and Ki-67 double-positive proliferating stem cells $(\sim 6.5 \%, \sim 1.5 \%, \sim 1 \%, \sim 1 \%$, and $\sim 0.9 \%$ at $\mathrm{P} 1,2$ weeks, 4 weeks, 12 weeks, and 24 weeks, respectively) with mouse age (Fig. 1B, C, Supplementary Table 1).

\section{SGNs contain self-renewing and proliferative activity}

Subsequently, to compare the biological significance of the SGN-NSCs derived from the cochlea of mice of different ages, we examined their self-renewing capacity by performing a sphere-forming assay in which the dissociated SGN-NSCs formed spheres at 1, 3, and 7 days in a serum-free medium (Fig. 2A). The dissociated cells from the primary spheres were grown further to form secondary spheres which showed self-renewing ability. The size and number of spheres in the P1 mice SGNNSCs increased significantly at 1, 3, and 7 days although this activity decreased critically in the 2- and 4-week mice SGNNSCs compared to the P1 mice SGN-NSCs (Fig. 2A, B). Moreover, to assess the stemness and proliferation of the SGN-NSCs, we successfully cultured both the P1 and P2-week-old mice SGN-NSCs in the presence of bFGF, but the number of SGNNSCs from P4-week-old mice was not enough for immunocytochemical analysis. Sox2-positive populations were highly expressed ( $~ 80 \%$ and $\sim 60 \%$ in the P1 and P2-week-old mice SGN-NSCs, respectively) and significantly decreased in both sets of cells over the course of differentiation (Fig. 2C, D). We also observed a high percentage of Nestin and Ki67 double-positive cells ( $45 \%$ of SGN-NSCs in the P1 mice and $\sim 35 \%$ in the P2-week-old mice) which significantly decreased over the course of differentiation in both sets of cells.

\section{SGNs contain multipotent differentiation potentials}

We also tested the differentiation potential of the SGN-NSCs in mice of different ages by observing their morphological changes (Fig. 3A). Upon induction of differentiation, the neurite outgrowth of the SGN-NSCs increased significantly (Fig. 3A). Fur-
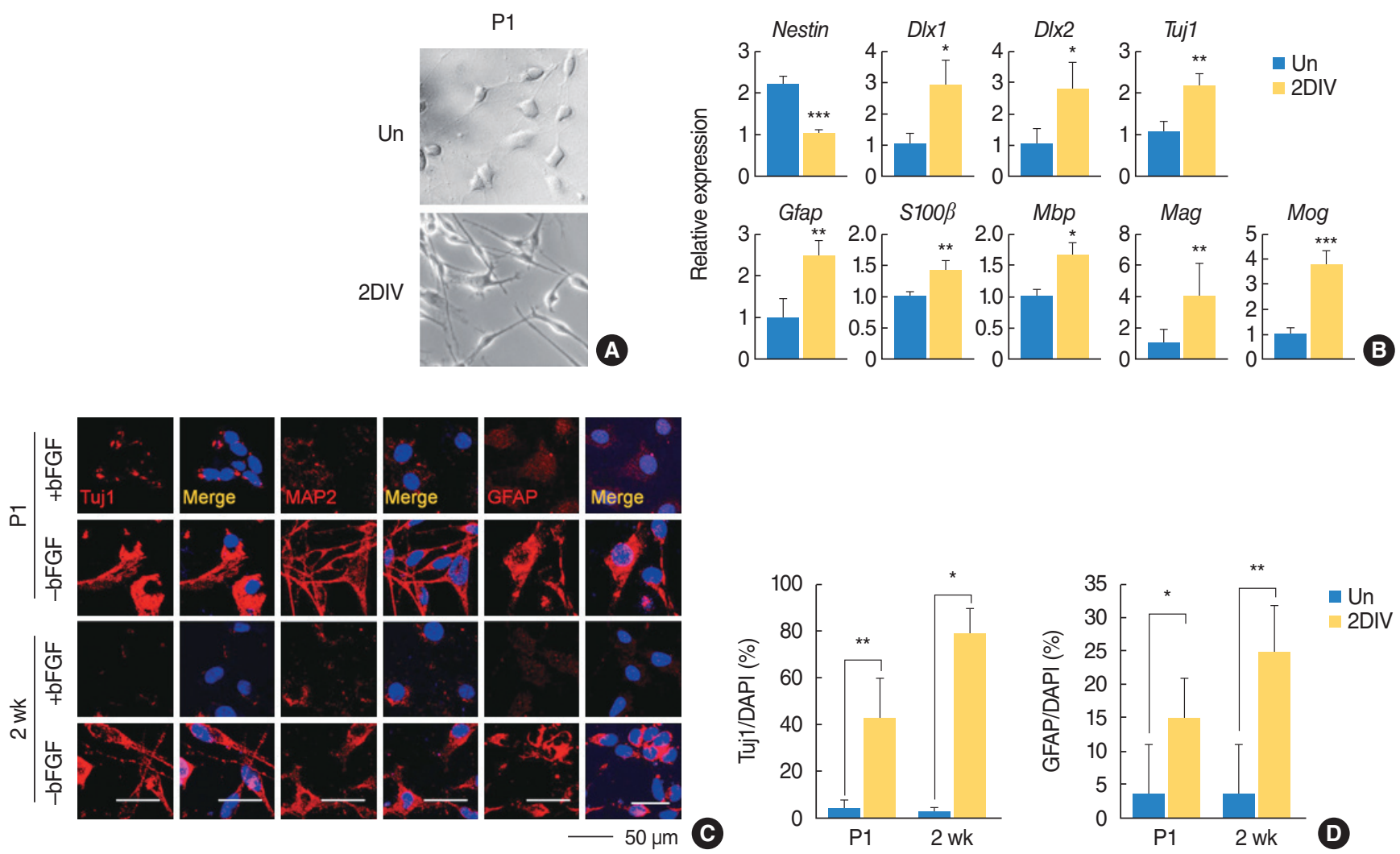

Fig. 3. The spiral ganglion cells exhibited a multipotent capacity. (A) Spiral ganglions were grown and their differentiation capabilities monitored by changes in morphology. (B) Quantitative polymerase chain reaction analysis of the indicated mRNA sequences in the postnatal day 1 (P1) mice spiral ganglions $(n=4)$. Values are presented as mean \pm standard deviation. $(C)$ Immunostaining with Tuj1, MAP2, and GFAP (all red) antibodies in $\mathrm{P1}$ and 2-week-old mice under undifferentiation and differentiation conditions. (D) Quantification of Tuj1-positive (P1: +bFGF, n=3; -bFGF, $n=5$; 2 weeks: +bFGF, n=3; -bFGF, $n=4$ ) and GFAP-positive cells (P1: +bFGF, n=5; -bFGF, $n=5 ; 2$ weeks: +bFGF, $n=4 ;-b F G F, n=4$ ) in (C). The $t$-tests were conducted to calculate the statistical significance $\left({ }^{\star} P<0.05,{ }^{* \star} P<0.005,{ }^{\star \star \star} P<0.0005\right)$. Un, undifferentiated; 2 DIV, 2 days differentiation in vitro; bFGF, basic fibroblast growth factor; MAP2, mature microtubule-associated protein 2; DAPI, 4',6-diamidino-2-phenylindole. 

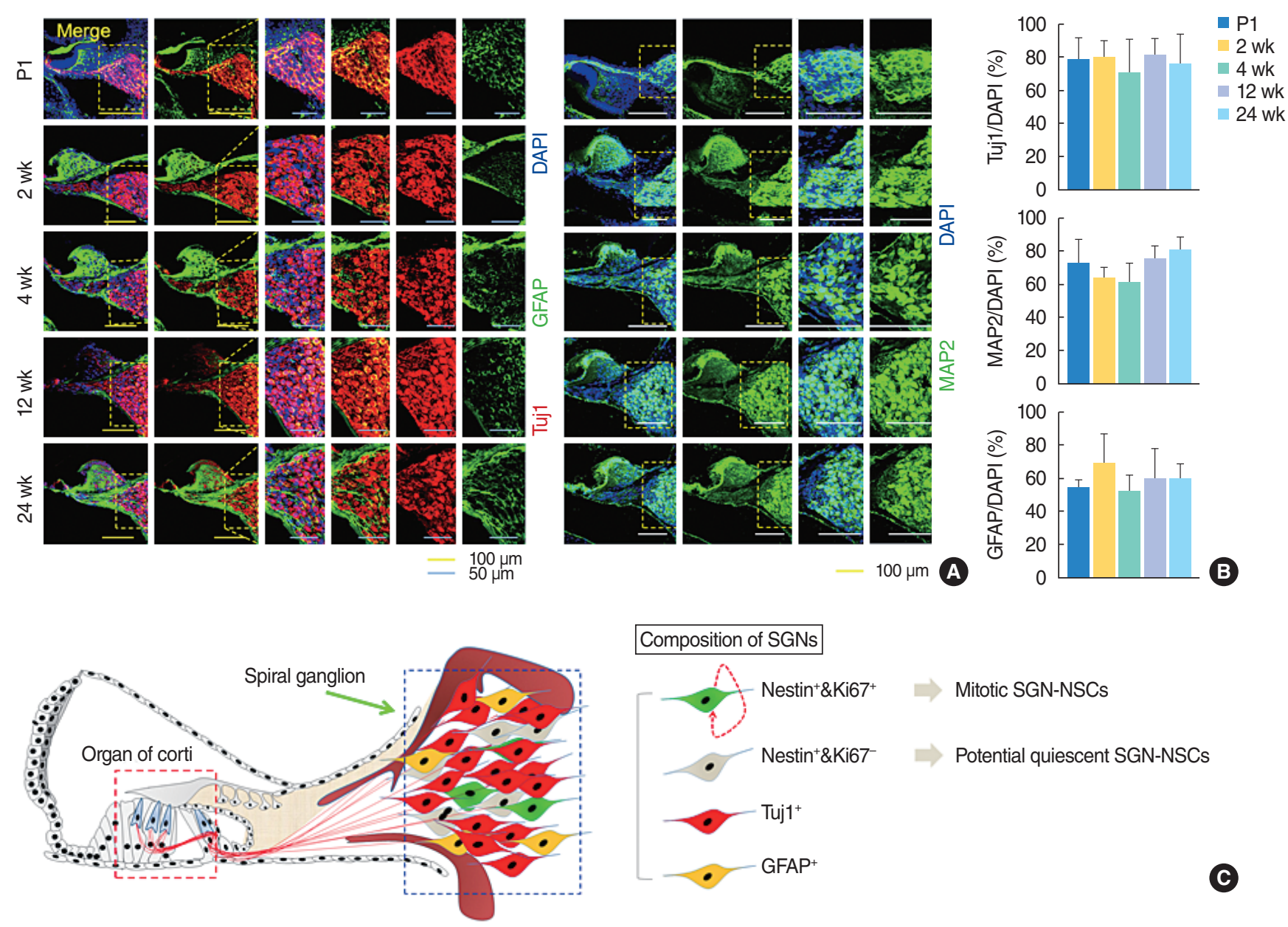

Composition of SGNs

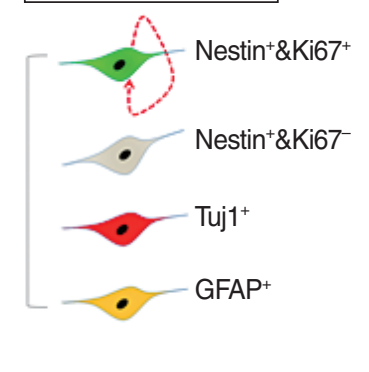

Mitotic SGN-NSCs

Potential quiescent SGN-NSCs

Fig. 4. Spiral ganglion cells consist of heterogeneous cell populations, of which spiral ganglion neuron-derived neural stem cell spheres (SGNNSCs) could be a regenerative source for damaged cell repair. (A) Fixed coronal sections of the cochlea stained with antibodies against GFAP (green), Tuj1 (red), and MAP2 (green). (B) Quantification of Tuj1-positive (postnatal day 1 [P1], n=4; 2 weeks, n=4; 4 weeks, n=3; 12 weeks, $n=4 ; 24$ weeks, $n=4)$, MAP2-positive (P1, $n=4 ; 2$ weeks, $n=4 ; 4$ weeks, $n=4 ; 12$ weeks, $n=4 ; 24$ weeks, $n=4)$, and GFAP-positive (P1, $n=4 ; 2$ weeks, $n=4 ; 4$ weeks, $n=3 ; 12$ weeks, $n=4 ; 24$ weeks, $n=4)$, in (A). (C) Schematic showing the existence of regenerative and self-renewing SGN-NSCs. DAPI, 4',6-diamidino-2-phenylindole; MAP2, mature microtubule-associated protein 2.

ther analysis using qPCR showed that the SGN-NSCs exhibited increased expression of $D l \times 1, D l \times 2$, and Tuj1 (markers for neuronal lineage) as well as other neural differentiation genes such as Gfap, $S 100 \beta$ (an astrocyte marker), Mbp, Mog, and Mag (the latter being an oligodendrocyte marker), and decreased expression of Nestin (Fig. 3B). Moreover, an immunocytochemical analysis of the P1 and P2-week-old mice SGN-NSCs revealed an increase in the number of cells positive for Tuj1, mature microtubule-associated protein 2 (MAP2), or GFAP (Fig. 3C, D). These results indicate that the SGN-NSCs attained a multipotent capacity.

The spiral ganglion consists of heterogeneous populations and the SGN-NSCs are a regenerative source for repairing damaged cells

To characterize the neural connections and glial cell composition in the spiral ganglion modiolus further, we undertook an immunohistochemical analysis of the cochlear tissues derived from the mice of different ages and observed the number of immature Tuj1-positive and mature MAP2-positive neurons, or GFAP-positive astrocytes did not significantly change in the mice of different ages (Fig. 4A, B). Overall, the spiral ganglion was heterogeneous and consisted of multiple populations of neuron, glial, non-proliferative SGNs, and proliferative SGNNSCs which exhibited both self-renewing activity and a multipotent capacity (Fig. 4C).

\section{DISCUSSION}

The existence of SGN-NSCs with stem cell-like properties have been confirmed in culture systems in vitro using ganglion cells derived from the cochlear modiolus of adult mice, but it is unclear how many SGN-NSCs were present and their age dependency 
in vivo. Given the significance of human SGN-NSCs as a potential source for regeneration and restoring hearing loss after cochlear implantation, further investigation was necessary to show the number of SGN-NSCs present in mice of different ages.

We observed significant decreases in Nestin and Ki-67 double-positive proliferating cells $(\sim 6.5 \%, \sim 1.5 \%, \sim 1 \%, \sim 1 \%$, and $\sim 0.9 \%$ at P1, 2 weeks, 4 weeks, 12 weeks, and 24 weeks, respectively) in vivo as a function of mouse age. Moreover, we observed a higher percentage of cells positive for the NSC marker Nestin $(\sim 52 \%, \sim 32 \%, \sim 27 \%, \sim 20 \%$, and $\sim 9 \%$ at P1, 2 weeks, 4 weeks, 12 weeks, and 24 weeks, respectively). These results demonstrate that in mice of different ages, the number of Nestin-positive SGN-NSCs was maintained to some extent, while the number of proliferating SGN-NSCs decreased, suggesting that a reduction in the regenerative ability in older adults compared to younger adults may be associated with a decrease in proliferating SGN-NSCs. Further, Nestin-positive and Ki67-negative cells need to be further characterized for their quiescent NSC property and potential to re-enter cell cycle. If they retain the ability to re-enter cell cycle and form functional SGNs, the availability of $9 \%$ Nestin-positive cells towards late age of 24 weeks holds huge promise for regeneration even in adults.

Our findings demonstrated the presence of SGN-NSCs in the mouse cochlear modiolus both in vitro and in vivo. Nestin-positive SGN-NSCs grew as neurospheres over time in culture and proliferated, as shown by the Ki67 labeling and the subculturing of the spheres. Although mammalian SGNs do not exhibit significant regenerative capacity, several groups have demonstrated that growth factor responsive progenitor/stem cells that proliferate and differentiate into mature neurons and glia cells could be isolated from the SGNs even in old adults $[6,8,9]$, which is in agreement with our findings. Furthermore, it has been reported that mitotic cells were found by means of the BrdU (5-bromo2'-deoxyuridine) incorporation method postnatally (P14) [14], although it was reported that the mitosis of mouse SGNs has mainly completed by gestational day 16 (E16) [15]. Our results also showed the presence of a few number of proliferated cells in mice of ages 12 weeks and older as indicated by Ki-67 marker. It was reported that Ki-67 antigen is expressed in all cycling cells, whereas BrdU reflects only the S-phage cells [16]. Importantly, the amount of proliferative stem cell populations shown in the P1 and 2-week-old mice SGN-NSCs were higher than those seen in the 4-, 12-, and 24-week-old mice SGN-NSCs, which supports the idea that the SGN-NSC spheres derived at an early age in mice contain more mitotic stem cells than those in mice at a later age. In immunohistochemical analysis with cochlear tissues derived from 12- and 24-week-old mice, we observed proliferative Nestin and Ki-67 double positive cells, but we couldn't succeed to grow SGN-NSCs and their spheres in vitro analysis. This might be due to low number of proliferative SGN-NSC population in old mice and further studies should be addressed. The inability to increase sphere size and number with age suggests that cells would potentially be entering a quiescent cell state due to intrinsic properties of stem cell to create a reserve pool once organ development is complete. Further studies are required to identify extrinsic molecules that can rewire this intrinsic cell property and allow the stem cells to proliferate in a homeostatic manner.

In our experiments, cell-type-specific antibodies showed their multipotency by the presence of Tuj1-positive early neurons, MAP2-positive matured neurons, and GFAP-positive astrocytes, which is similar to previous reports [8,9]. In our SGN-NSCs cultures, we observed Nestin-positive NSC populations that divided over time, as shown by the Ki-67 staining and the secondary subcultivation of the neurospheres. These cells subsequently differentiated into neurons in the absence of bFGF. Therefore, our conclusion is that the SGN dividing cells, rather than being differentiated mature neurons or glial cells, represent proliferating NSCs that exist in the mature spiral ganglia of the VIII cranial nerve.

Previous mouse studies have revealed the existence of sphereforming SGN-NSCs from embryonic day 12.5 to postnatal day $5[6,7,10]$. In another study, the spiral ganglion was shown to possess a high proliferative potential after birth but to lose the vast majority of stem cells steeply between the second and third postnatal week, and to show no proliferative activity at P42 [8]. It has been reported that SGN-NSCs exist even in human adults aged between 40 and 60 years old and develop into the form of spheres that proliferate and differentiate into mature neurons and glial cells [9], suggesting that these cells could be of importance for the self-repair, self-renewing, and maintenance of the functional integrity of the auditory nerve throughout life [17]. We observed that the proliferative potential of the SGN-NSCs in mice persisted up to postnatal 3-6 months in vivo, albeit at a low level, suggesting that they might serve as donor cells in stem cell-based SGN replacement therapy, even at a later age in the animals' lives.

SGNs are an essential functional component of the peripheral auditory system. Most types of hearing loss are associated with spiral ganglion cell degeneration, which is irreversible due to the inner ear's lack of regenerative capacity. Although transplantation of exogenous stem cells to replace or maintain decreased SGNs has been pursued to restore hearing function [4,18-20], the lack of functional and survived donor cells after implantation of cells are major issues to the successful implementation of therapeutic transplantation [6]. Another option is to recruit new SGNs from endogenous cell sources with functional connections in the auditory system under appropriate growth factor stimulation [3]. If there are NSCs in the SGN region, the lost SGNs might be replaced by NSCs. It has been reported that embryonic stem cells and SGNs from newborn mice make synapses with hair cells and contact neurons in the brainstem [4,7,21]. Poor prognostic factors for CI outcomes, such as old age and a long duration of deafness, in postlingually deaf adults are possibly related to a decrease in SGN count, which leaves fewer neurons 
available for stimulation by the $\mathrm{CI}$, even though central re-organization might also play a role in determining the $\mathrm{CI}$ outcomes [22-24]. The advantage of CI on the survival of SGNs is that electrical stimulation could enhance their survival and a combination of induced expression of glial cell line-derived neurotrophic factor in the cochlea using viral vectors and electrical stimulation has also been shown to have a synergic effect on increasing the survival of SGNs [25,26], leading to the better survival or regeneration of SGNs from endogenous NSCs in patients with a CI for hearing restoration.

In summary, we found the presence of sphere-forming stem cells isolated from the spiral ganglion of postnatal mice both in vivo and in vitro, although the proliferating activity decreased significantly with age. We showed that spiral ganglion spheres have characteristics similar to neurospheres isolated from the brain and maintained their major stem cell characteristics after repeated propagation [11-13], which enabled the culturing of spheres over an extended period of time. The SGN-NSCs could differentiate into several cell populations, including neurons and glial cells. Thus, our work provides evidence for the presence of self-renewing spiral ganglion stem cells which might serve as a promising source for the regeneration of lost auditory neurons.

\section{CONFLICT OF INTEREST}

No potential conflict of interest relevant to this article was reported.

\section{SUPPLEMENTARY MATERIALS}

Supplementary materials can be found via https://doi.org/10. 21053/ceo.2018.00878.

\section{REFERENCES}

1. Kujawa SG, Liberman MC. Adding insult to injury: cochlear nerve degeneration after "temporary" noise-induced hearing loss. J Neurosci. 2009 Nov;29(45):14077-85.

2. Hoeffding V, Feldman ML. Changes with age in the morphology of the cochlear nerve in rats: light microscopy. J Comp Neurol. 1988 Oct;276(4):537-46.

3. Roehm PC, Hansen MR. Strategies to preserve or regenerate spiral ganglion neurons. Curr Opin Otolaryngol Head Neck Surg. 2005 Oct;13(5):294-300.

4. Shi F, Edge AS. Prospects for replacement of auditory neurons by stem cells. Hear Res. 2013 Mar;297:106-12.

5. Reyes JH, O'Shea KS,Wys NL,Velkey JM, Prieskorn DM, Wesolowski K, et al. Glutamatergic neuronal differentiation of mouse embryonic stem cells after transient expression of neurogenin 1 and treatment with BDNF and GDNF: in vitro and in vivo studies. J Neurosci. 2008 Nov;28(48):12622-31.

6. Zhang L, Jiang H, Hu Z. Concentration-dependent effect of nerve growth factor on cell fate determination of neural progenitors. Stem Cells Dev. 2011 Oct;20(10):1723-31.

7. Li X, Aleardi A, Wang J, Zhou Y, Andrade R, Hu Z. Differentiation of spiral ganglion-derived neural stem cells into functional synaptogenetic neurons. Stem Cells Dev. 2016 May;25(10):803-13.

8. Oshima K, Grimm CM, Corrales CE, Senn P, Martinez Monedero R, Geleoc GS, et al. Differential distribution of stem cells in the auditory and vestibular organs of the inner ear. J Assoc Res Otolaryngol. 2007 Mar;8(1):18-31.

9. Rask-Andersen H, Bostrom M, Gerdin B, Kinnefors A, Nyberg G, Engstrand T, et al. Regeneration of human auditory nerve: in vitro/ in video demonstration of neural progenitor cells in adult human and guinea pig spiral ganglion. Hear Res. 2005 May;203(1-2):180-91.

10. Diensthuber M, Zecha V, Wagenblast J, Arnhold S, Stover T. Clonal colony formation from spiral ganglion stem cells. Neuroreport. 2014 Oct;25(14):1129-35.

11. Moon BS, Bai J, Cai M, Liu C, Shi J, LuW. Kruppel-like factor 4-dependent Staufen1-mediated mRNA decay regulates cortical neurogenesis. Nat Commun. 2018 Jan;9(1):401.

12. Moon BS, Kim HY, Kim MY,Yang DH, Lee JM, Cho KW, et al. Sur8/ Shoc2 involves both inhibition of differentiation and maintenance of self-renewal of neural progenitor cells via modulation of extracellular signal-regulated kinase signaling. Stem Cells. 2011 Feb;29(2): 320-31.

13. Moon BS, Yun HM, Chang WH, Steele BH, Cai M, Choi SH, et al. Smek promotes corticogenesis through regulating Mbd3's stability and Mbd3/NuRD complex recruitment to genes associated with neurogenesis. PLoS Biol. 2017 May;15(5):e2001220.

14. Kamiya K, Takahashi K, Kitamura K, MomoiT, Yoshikawa Y. Mitosis and apoptosis in postnatal auditory system of the $\mathrm{C} 3 \mathrm{H} / \mathrm{He}$ strain. Brain Res. 2001 May;901(1-2):296-302.

15. Ruben RJ. Development of the inner ear of the mouse: a radioautographic study of terminal mitoses. Acta Otolaryngol. 1967:Suppl 220: $1-44$.

16. Tinnemans MM, Lenders MH, ten Velde GP, Wagenaar SS, Blijham $\mathrm{GH}$, Ramaekers FC, et al. Evaluation of proliferation parameters in in vivo bromodeoxyuridine labelled lung cancers. Virchows Arch. 1995;427(3):295-301.

17. Kempermann G, Gage FH, Aigner L, Song H, Curtis MA, Thuret S, et al. Human adult neurogenesis: evidence and remaining questions. Cell Stem Cell. 2018 Jul;23(1):25-30.

18. Tamura T, Nakagawa T, Iguchi F, Tateya I, EndoT, Kim TS, et al.Transplantation of neural stem cells into the modiolus of mouse cochleae injured by cisplatin. Acta Otolaryngol Suppl. 2004 Mar;(551):65-8.

19. Hu Z, Andang M, Ni D, Ulfendahl M. Neural cograft stimulates the survival and differentiation of embryonic stem cells in the adult mammalian auditory system. Brain Res. 2005 Jul;1051(1-2):137-44.

20. Zhang Y, Pak C, Han Y, Ahlenius H, Zhang Z, Chanda S, et al. Rapid single-step induction of functional neurons from human pluripotent stem cells. Neuron. 2013 Jun;78(5):785-98.

21. Vierbuchen T, Ostermeier A, Pang ZP, Kokubu Y, Sudhof TC, Wernig M. Direct conversion of fibroblasts to functional neurons by defined factors. Nature. 2010 Feb;463(7284):1035-41.

22. Khan AM, Handzel O, Burgess BJ, Damian D, Eddington DK, Nadol JB Jr. Is word recognition correlated with the number of surviving spiral ganglion cells and electrode insertion depth in human subjects with cochlear implants? Laryngoscope. 2005 Apr;115(4):672-7.

23. Fayad JN, Linthicum FH Jr. Multichannel cochlear implants: relation of histopathology to performance. Laryngoscope. 2006 Aug;116(8): 1310-20.

24. Nadol JB Jr, Young YS, Glynn RJ. Survival of spiral ganglion cells in profound sensorineural hearing loss: implications for cochlear implantation. Ann Otol Rhinol Laryngol. 1989 Jun;98(6):411-6.

25. Lousteau RJ. Increased spiral ganglion cell survival in electrically 
stimulated, deafened guinea pig cochleae. Laryngoscope. $1987 \mathrm{Jul}$; 97(7 Pt 1):836-42.

26. Kanzaki S, Stover T, Kawamoto K, Prieskorn DM, Altschuler RA,
Miller JM, et al. Glial cell line-derived neurotrophic factor and chronic electrical stimulation prevent VIII cranial nerve degeneration following denervation. J Comp Neurol. 2002 Dec;454(3):350-60. 